\title{
Investigation of candidate polymorphisms and disease activity in rheumatoid arthritis patients on methotrexate
}

\author{
Yvonne C. Lee ${ }^{1}$, Jing Cui ${ }^{1}$, Karen H. Costenbader ${ }^{1}$, Nancy A. Shadick ${ }^{1}$, Michael E. Weinblatt ${ }^{1}$ \\ and Elizabeth W. Karlson ${ }^{1}$
}

\begin{abstract}
Objectives. We examined the association between candidate single nucleotide polymorphisms (SNPs) and disease activity in RA patients on MTX.

Methods. Our population was drawn from the Brigham and Women's Hospital Rheumatoid Arthritis Sequential Study (BRASS), a prospective, observational cohort of RA patients. A total of 556 participants were genotyped using the Affymetrix $100 \mathrm{~K}$ platform. Two hundred and sixty-two participants were on MTX therapy, including 120 on MTX monotherapy. The primary outcome was the disease activity score in 28 joints (DAS28-CRP). High disease activity was defined as DAS28-CRP $>3.2$. Low disease activity was defined as DAS28CRP $\leqslant 3.2$. We studied three candidate alleles in the ATIC, ITPA and MTHFR genes for association with DAS28-CRP.

Results. Among participants on MTX monotherapy, those carrying the minor allele of ATIC SNP rs4673993 were more likely to have low disease activity $(P=0.01)$. None of the other SNPs was associated with disease activity. Among patients on any MTX (combination or monotherapy), the minor allele of $A T I C$ rs4673993 was also associated with low disease activity $(P=0.04)$.

Conclusions. In this cross-sectional analysis, ATIC SNP rs4673993 was associated with low disease activity in patients on MTX. Further studies are needed to clarify the relationship between ATIC polymorphisms, disease activity and treatment response.
\end{abstract}

Key words: MTX, Adenosine, RA, Disease activity.

\section{Introduction}

MTX is the most popular drug worldwide for the treatment of RA, but many patients do not respond adequately, requiring the addition of other medications or a switch to another diseasemodifying agent $[1,2]$. Despite its common use, MTX's mechanism of action is not well understood, and predictors of treatment response have not been fully characterized.

Candidate gene studies have been used to gain greater insight into MTX's mechanism of action and to discover genetic predictors of treatment response. Studies have suggested an association between MTX treatment response and several genes involved in MTX metabolism and pathways of inflammation. These genes have included 5-aminoimidazole-4-carboxamide ribonucleotide formyltransferase/IMP cyclohydrolase (ATIC) [3-5], inosine triphosphate phosphorylase $(I T P A)$ [4, 5] and 5,10methylenetetrahydrofolate reductase (MTHFR) [6-8].

$A T I C$ and ITPA are genes in the adenosine pathway. They encode enzymes involved in the release of extracellular adenosine, a molecule with anti-inflammatory properties [4]. MTHFR is a gene in the homocysteine pathway. It encodes an enzyme involved in the generation of homocysteine, an amino acid associated with the pro-inflammatory response [8].

Although multiple candidate gene studies have been performed, the true association between these genes and MTX treatment response is still unclear. Many of these studies were small, and results have not been consistently replicated.

In this study, we examined the association between disease activity in RA patients on MTX and SNPs previously implicated to have an association with MTX treatment response. We hypothesized that low disease activity would be associated with the

'Division of Rheumatology, Immunology and Allergy, Brigham and Women's Hospital and Harvard Medical School, Boston, MA, USA.

Submitted 2 September 2008; revised version accepted 19 December 2008.

Correspondence to: Yvonne C. Lee, Division of Rheumatology, Immunology and Allergy, 75 Francis Street, PBB-B3, Boston, MA 02115, USA.

E-mail: ylee9@partners.org major allele of $A T I C$ rs4673993 [in linkage disequilibrium (LD) with $A T I C$ rs2372536, $\left.r^{2}=0.96\right]$, the major allele of ITPA rs1127354 and the minor allele of MTHFR rs1801133.

\section{Methods}

\section{Study population and data collection}

The participants in this study are a subset of the 984 participants in the Brigham and Women's Hospital Rheumatoid Arthritis Sequential Study (BRASS), a prospective, observational, hospitalbased cohort of individuals with established RA, recruited for the identification of biomarkers and genetic predictors of drug response and disease activity. Annual examinations included an assessment of medication changes and joint counts by physicians. Patients also completed self-administered surveys, such as the Multi-Dimensional HAQ (MDHAQ). Blood samples for immunophenotyping, including CRP, and genetic testing were collected at baseline and annually. The data used in this study consist of information collected from the baseline visit. The Partners Institutional Review Board approved the study, and written informed consent was obtained from all participants. Additional details can be found in a past publication [9].

For the primary analysis, we included the 120 participants taking MTX monotherapy (thus excluding those taking concurrent anti-TNF medications) for at least 2 months prior to the baseline BRASS visit. For secondary analyses, we expanded the population to include a total of 262 participants on any MTX therapy (monotherapy or combination therapy). This included the original 120 participants and an additional 142 patients on MTX therapy combined with another DMARD or anti-TNF agent. For SNPs associated with disease activity in the primary analysis and/ or secondary analysis, we also performed post hoc analyses on the subgroup of patients on combination therapy with MTX and an anti-TNF agent/DMARD.

\section{Clinical predictors}

Potential clinical predictors of disease activity were chosen based on the literature $[5,10]$. For the primary analysis, involving participants on MTX monotherapy, clinical predictors included age, sex, RA disease duration, cigarette smoking status (non-smoker, 
current smoker), RF status, anti-cyclic citrullinated peptide (CCP) status, prior DMARD use, prior anti-TNF use, MDHAQ score, duration of MTX therapy and dose of MTX. For secondary analyses, involving all participants on MTX, concurrent DMARD use and concurrent anti-TNF use were also assessed as potential predictors of disease activity. Age was analysed as a continuous variable. Due to non-linearity, disease duration, MDHAQ score, duration of MTX therapy and dose of MTX were categorized into groups based on median split or quantiles.

\section{Genetic predictors}

Five hundred and fifty-six of the BRASS participants were previously genotyped using the Affymetrix 100K platform [11]. Non-Caucasian participants $(n=10)$ were excluded because allele frequencies differ between races and can be a source of bias. To choose candidate SNPs, we searched PubMed for other candidate gene studies of MTX treatment response, using the search terms, 'Methotrexate', 'Arthritis, Rheumatoid/drug therapy' and 'Arthritis, Rheumatoid/genetics'. To be included in this study, candidate SNPs had to be associated $(P<0.05)$ with MTX treatment response in at least one prior study that included at least 100 RA patients. The seven SNPs that met these criteria were ATIC rs2372536 $(347 C / G)$ [3-5], ITPA rs1127354 (94C/A) [4, 5], MTHFR rs1801133 (677C/T) [6-8], MTHFR $\mathrm{rs} 1801131$ (1298A/C) [8], RFC-1/SLC19A1 rs1051266 (80G/A) [3], MDR1/ $A B C B 1$ rs $1045642(3435 \mathrm{C} / \mathrm{T}) \quad[12,13]$ and $T N F \alpha$ rs361525 $(-235 \mathrm{~A} / \mathrm{G})[14]$.

Of these SNPs, only ITPA $94 \mathrm{C} / \mathrm{A}$ (rs1127354) and MTHFR 677C/T (rs1801133) were included on the Affymetrix 100K chip. For the remaining SNPs, we chose appropriate proxy SNPs $\left(r^{2}>0.8\right)$ on the Affymetrix $100 \mathrm{~K}$ chip. If SNPs did not have a reasonable proxy $\left(r^{2}>0.8\right)$, we excluded them from the analysis. The final list of SNPs included ATIC rs4673993 $\left(r^{2}=0.96\right.$ with ATIC rs2372536), ITPA rs1127354 and MTHFR rs1801133. Hardy-Weinberg equilibrium was assessed for each SNP.

\section{Outcomes}

The primary outcome for both analyses was the disease activity score in 28 joints (DAS28-CRP) at the BRASS baseline visit. Low disease activity was defined as DAS28-CRP $\leqslant 3.2$. Moderate/high disease activity was defined as a DAS28-CRP $>3.2[15]$.

\section{Statistical testing}

For categorical variables, the univariate association between potential genetic predictors, clinical predictors and disease activity was assessed using Fisher's exact tests and chi-square tests. A $t$-test was used to assess the association between the continuous variable age and disease activity. Collinearity between predictors was assessed using Spearman's correlation. Predictors correlated with each other with $P<0.005$ were considered collinear, and only the most significant predictor was considered for inclusion in multivariable logistic regression analyses. All non-collinear predictors associated with outcome at $P<0.15$ were retained for multivariable analyses.

To examine the likelihood that our results were false-positive findings, we calculated the false-positive report probability (FPRP) using the methods described by Wacholder et al. [16]. We set 0.5 as the FPRP cut-off for a noteworthy value. The expected odds ratios (ORs) were based on reported ORs from previous studies [5]. Given the previous literature suggesting associations between these polymorphisms and MTX treatment response, we set the prior probability of an association between each SNP and MTX treatment response at 0.1. A prior probability of 0.1 represents a moderate to high prior probability of association and has been used in studies involving candidate genes/SNPs with prior evidence of association with disease [17-20].

\section{Results}

A total of 120 participants were on MTX monotherapy, and 142 participants were on combination therapy that included MTX. Eighty-five percent were female. Mean age was 59 yrs with an average disease duration of $17 \mathrm{yrs}$. Detailed information regarding baseline characteristics is presented in Table 1. All SNPs were in Hardy-Weinberg equilibrium in the primary and secondary analyses populations.

\section{MTX monotherapy}

Among participants on MTX monotherapy, 35 (29\%) had low disease activity, and $85(71 \%)$ had moderate/high disease activity. In univariate analyses, younger age, shorter disease duration and negative anti-CCP status were associated with low disease activity at $P<0.05$ (Table 2). Age was correlated with disease duration at $P<0.005$; therefore, age was not included in the multivariable model.

The minor allele of $A T I C$ rs4673993 was associated with low disease activity in patients on MTX monotherapy $(P=0.006$; OR 3.86; 95\% CI 1.50, 9.91). ITPA $94 \mathrm{C} / \mathrm{A}$ (rs1127354) and MTHFR $677 \mathrm{C} / \mathrm{T}$ (rs1801133) were not associated with disease activity (Table 3). After adjustment for disease duration and anti-CCP status in multivariable analyses, the minor allele of $A T I C$ rs4673993 remained significantly associated with low disease activity in patients on MTX monotherapy $(P=0.01$, OR 3.89; $95 \%$ CI 1.36, 11.14) (Table 4). Based on a moderate prior probability of 0.1 and an expected OR of 2.5 , the FPRP for an association between ATIC rs4673993 and disease activity was 0.33 . For prior probabilities ranging between 0.01 and 0.1 and ORs ranging between 1.5 and 3.5, the FPRP varied from 0.10 to 0.97 (Table 5).

TABLE 1. Baseline characteristics of participants on MTX monotherapy, MTX combination therapy and any MTX therapy (monotherapy or combination therapy)

\begin{tabular}{|c|c|c|c|}
\hline Baseline variables & $\begin{array}{l}\text { MTX monotherapy } \\
\qquad(n=120)\end{array}$ & $\begin{array}{l}\text { MTX combination therapy } \\
\qquad(n=142)\end{array}$ & $\begin{array}{l}\text { Any MTX } \\
(n=262)\end{array}$ \\
\hline Age, mean (s.D.), yrs & $61.5(12.6)$ & $56.9(12.3)$ & $59.0(12.6)$ \\
\hline Gender (female), $n(\%)$ & $103(85.8)$ & $120(84.5)$ & $223(85.1)$ \\
\hline Disease duration, mean (s.D.), yrs & $18.1(13.0)$ & $16.4(10.7)$ & $17.2(11.8)$ \\
\hline RF positive, $n(\%)$ & $95(79.2)$ & $114(80.3)$ & $209(79.8)$ \\
\hline Anti-CCP positive, $n(\%)$ & $86(72.9)$ & $114(80.3)$ & $200(76.9)$ \\
\hline Current smokers, $n(\%)$ & $8(7.2)$ & $11(8.5)$ & $19(7.9)$ \\
\hline MDHAQ score, mean (s.D.) & $0.7(0.5)$ & $0.7(0.5)$ & $0.7(0.5)$ \\
\hline Duration of MTX treatment, mean (S.D.), months & $74.2(77.1)$ & $62.1(53.8)$ & $67.6(65.7)$ \\
\hline MTX dose, mean (s.D.), mg & $13.0(7.2)$ & $12.8(6.9)$ & $12.9(7.0)$ \\
\hline Concurrent DMARD users, $n(\%)$ & NA & $52(36.6)$ & $52(19.9)$ \\
\hline Concurrent anti-TNF users, $n(\%)$ & NA & $96(67.6)$ & $96(36.6)$ \\
\hline Past DMARD users, $n(\%)$ & $91(75.8)$ & $105(73.9)$ & $196(74.8)$ \\
\hline Past anti-TNF users, $n(\%)$ & $12(10.0)$ & $29(20.4)$ & $41(15.7)$ \\
\hline
\end{tabular}


TABLE 2. Univariate association between clinical predictors of low disease activity (defined by baseline DAS28-CRP $\leqslant 3.2$ ) in participants on MTX monotherapy

\begin{tabular}{|c|c|c|c|c|}
\hline Baseline variables & $\begin{array}{c}\text { DAS28-CRP } \leqslant 3.2 \\
(n=35)\end{array}$ & $\begin{array}{c}\text { DAS28-CRP }>3.2 \\
(n=85)\end{array}$ & $\begin{array}{c}\text { OR } \\
(95 \% \mathrm{Cl})\end{array}$ & $P$-value \\
\hline Age, mean (s.D.), yrs & $55.3(14.4)$ & $63.9(10.9)$ & - & 0.003 \\
\hline Gender (female), $n(\%)$ & $29(82.9)$ & $74(87.1)$ & $0.72(0.24,2.12)$ & 0.57 \\
\hline Current smokers, $n(\%)$ & $3(9.7)$ & $5(6.3)$ & $1.61(0.36,7.17)$ & 0.68 \\
\hline RF positive, $n(\%)$ & $26(74.3)$ & $69(79.8)$ & $0.67(0.26,1.70)$ & 0.46 \\
\hline Anti-CCP positive, $n(\%)$ & $19(55.9)$ & $67(79.8)$ & $0.32(0.14,0.76)$ & 0.01 \\
\hline Disease duration, mean (s.D.), yrs & $12.9(12.5)$ & $20.2(12.7)$ & $0.26(0.11,0.62)$ & 0.002 \\
\hline MDHAQ score, mean (s.D.) & $0.6(0.5)$ & $0.7(0.6)$ & - & 0.23 \\
\hline Duration of MTX treatment, mean (S.D.), months & $58.8(63.2)$ & $80.5(81.7)$ & - & 0.65 \\
\hline MTX dose, mean (s.D.), mg & $12.0(7.5)$ & $13.5(7.0)$ & - & 0.28 \\
\hline Past DMARD users, $n(\%)$ & $24(68.6)$ & $67(78.2)$ & $0.59(0.24,1.42)$ & 0.25 \\
\hline Past anti-TNF users, $n(\%)$ & $4(11.4)$ & $8(9.4)$ & $1.24(0.35,4.42)$ & 0.74 \\
\hline
\end{tabular}

TABLE 3. Univariate association between genotype and disease activity among participants on MTX monotherapy

\begin{tabular}{|c|c|c|c|c|c|}
\hline Gene & Genotype & $\begin{array}{l}\text { Genotype } \\
\text { counts (\%) }\end{array}$ & $\begin{array}{l}\text { Number with low } \\
\text { DAS28-CRP (\%) }\end{array}$ & $\begin{array}{c}\mathrm{OR}^{\mathrm{a}} \\
(95 \% \mathrm{Cl})\end{array}$ & $P$-value \\
\hline$A T I C$ & TT & $52(44.8)$ & 7 (13.5) & $3.86(1.50,9.91)$ & 0.006 \\
\hline \multirow[t]{2}{*}{ rs4673993 } & $\mathrm{TC}$ & $50(43.1)$ & $19(38.0)$ & & \\
\hline & $\mathrm{CC}$ & $14(12.1)$ & $5(35.7)$ & & \\
\hline ITPA & $\mathrm{CC}$ & $102(85.7)$ & $31(30.4)$ & $0.49(0.13,1.83)$ & 0.39 \\
\hline \multirow[t]{2}{*}{ rs1127354 } & $\mathrm{CA}$ & $16(13.5)$ & $3(18.8)$ & & \\
\hline & $\mathrm{AA}$ & $1(0.8)$ & $0(0)$ & & \\
\hline MTHFR & $\mathrm{CC}$ & $50(42.4)$ & $15(30.0)$ & $0.90(0.40,2.02)$ & 0.84 \\
\hline \multirow[t]{2}{*}{ rs1801131 } & CT & 53 (44.9) & $18(24.0)$ & & \\
\hline & TT & $15(12.7)$ & $1(6.7)$ & & \\
\hline
\end{tabular}

${ }^{\mathrm{a} O R}$ is for the association between the minor allele and low DAS28-CRP.

TABLE 4. Association between ATIC rs4673993 and low disease activity among MTX monotherapy, adjusted for disease duration and anti-CCP status

\begin{tabular}{llc}
\hline \multicolumn{1}{c}{ Variable } & OR $(95 \% \mathrm{Cl})$ & $P$-value \\
\hline ATIC rs4673993 & $3.89(1.36,11.14)$ & 0.01 \\
Disease duration & $0.23(0.08,0.62)$ & 0.004 \\
Anti-CCP status & $0.40(0.14,1.10)$ & 0.08 \\
\hline
\end{tabular}

TABLE 5. False positive report probability (FPRP) for the association between ATIC rs4673993 and disease activity among participants on MTX monotherapy, according to varying prior probabilities

\begin{tabular}{lccc}
\hline & \multicolumn{3}{c}{ OR } \\
\cline { 2 - 4 } Prior probability & 1.5 & 2.5 & 3.5 \\
\hline 0.20 & 0.55 & 0.18 & 0.10 \\
0.15 & 0.63 & 0.24 & 0.13 \\
0.10 & 0.73 & 0.33 & 0.19 \\
0.05 & 0.85 & 0.51 & 0.34 \\
0.01 & 0.97 & 0.85 & 0.73 \\
\hline
\end{tabular}

\section{Any MTX therapy}

Among participants on any MTX (monotherapy or combination therapy), 90 (34\%) had low disease activity, and 172 (66\%) had moderate/high disease activity. In univariate analyses, shorter disease duration, younger age, low MDHAQ score, negative antiCCP status and concurrent anti-TNF use were associated with low disease activity at $P<0.05$. Although not statistically significant, shorter duration of MTX treatment, male gender and past DMARD use were also associated with low disease activity at $P<0.15$ and were thus considered for inclusion in the final model. However, disease duration, anti-CCP status, concurrent anti-TNF use and gender were the only clinical variables that were not correlated with each other with $P<0.005$.

The minor allele of $A T I C$ rs4673993 was significantly associated with low disease activity $(P=0.03$; OR 1.81 ; $95 \%$ CI 1.05 , 3.10). None of the other SNPs was associated with disease activity.
After adjustment for the significant clinical variables in multivariable analyses, the minor allele of $A T I C$ rs4673993 remained significantly associated with low disease activity $(P=0.04$; OR $1.88 ; 95 \%$ CI 1.03, 3.43) (Table 6). Based on a moderate prior probability of 0.1 and an expected OR of 2.5, the FPRP for an association between ATIC rs4673993 and disease activity was 0.30 . The FPRP ranged from 0.14 for a prior probability of 0.2 and an OR of 3.5 to 0.94 for a prior probability of 0.01 and an OR of 1.5 .

\section{Post hoc analyses}

Among the 142 participants on combination therapy with MTX and an anti-TNF agent or DMARD, the minor allele of ATIC rs4673993 was not significantly associated with low disease activity $(P=0.86$; OR $1.13 ; 95 \%$ CI $0.56,2.25)$.

\section{Discussion}

In this cross-sectional study of 120 established RA patients on MTX monotherapy, the minor allele of ATIC rs4673993 was associated with low disease activity. ATIC rs4673993 is in an intronic region and does not code for protein. Introns are historically viewed as inert regions of DNA that function as 'spacers' between exons, but recent studies suggest that introns may play a role in the regulation of gene expression [21]. Alternatively, intronic SNPs, such as $A T I C$ rs4673993, may be in LD with other SNPs that change protein structure or alter gene expression.

Previous studies have suggested potential associations between $A T I C$ SNPs and treatment response [3-5]. In a cross-sectional study by Dervieux et al. of 108 RA patients on MTX for at least 3 months, ATIC 347 GG (rs2372536, minor genotype) was associated with better physician's assessment of treatment response $(P=0.02)$ than $A T I C 347 \mathrm{GC}$ and $\mathrm{CC}$ [3]. A second study by Wessels et al. [4] also showed an association between $A T I C$ rs2372536 and treatment response, but the direction of association was opposite to that seen in the initial study. This study of 205 RA patients reported that ATIC 347 CC (rs2372536, major genotype) was associated with good clinical response to MTX at 6 months. The authors hypothesized that the discrepancy between the results of these two studies may have been due to differences in study population (early onset $v s$ established RA) and study design (longitudinal, clinical trial data $v s$ cross-sectional study) [4].

The SNP examined in the present study, ATIC rs4673993, is in LD with $A T I C$ rs2372536, the non-synonymous coding SNP in the studies above [3-5]. Given our assumption that ATIC rs4673993 is a proxy for $A T I C$ rs2372536, our results are most consistent with the initial study in which the minor genotype was associated with lower disease activity [3]. Alternatively, ATIC rs4673993 may be in LD with another coding SNP that affects enzyme activity differently than the $A T I C$ rs2372536 polymorphism, or $A T I C$ rs4673993 may have a regulatory function that positively influences disease activity. 
TABLE 6. Association between ATIC rs4673993 and low disease activity among all MTX users, adjusted for disease duration, anti-CCP status, anti-TNF use and gender

\begin{tabular}{lcc}
\hline \multicolumn{1}{c}{ Variable } & OR $(95 \% \mathrm{Cl})$ & $P$-value \\
\hline ATIC rs4673993 & $1.88(1.03,3.43)$ & 0.04 \\
Disease duration & $0.25(0.14,0.44)$ & $<0.0001$ \\
Anti-CCP status & $0.41(0.20,0.81)$ & 0.01 \\
Anti-TNF use & $2.75(1.50,5.03)$ & 0.001 \\
Female gender & $0.53(0.24,1.17)$ & 0.12 \\
\hline
\end{tabular}

Our study population and study design also differed from the second study, providing another potential reason for the disparate results. Participants in our study had longer disease durations (mean 18 yrs vs 2 weeks) and were more likely to be RF positive (79.2 vs $67.3 \%$ ) compared with participants in the Wessels et al. study. Furthermore, we restricted our analyses to Caucasian participants, whereas Wessels et al. [4] included non-Caucasian participants. Wessels et al. [4] utilized data from a randomized clinical trial, whereas this study was a cross-sectional, observational study [4]. Our outcome was disease activity in patients on MTX rather than treatment response after 6 months of therapy.

The difference between our results and those of Wessels may also be explained by a false-positive report in either or both studies. Given the large number of genetic association tests performed, false-positive results are frequent. We did not adjust for multiple comparisons because corrective procedures may unnecessarily lower power [16]; instead, we assessed the likelihood of a falsepositive report using the FPRP.

To calculate the FPRP, we used a prior probability of 0.1 and an expected OR of 2.5. A 0.1 is considered a moderate to high prior probability. We believe this is justified based on prior reports of an association between ATIC SNPs and MTX treatment response $[3,5]$. The expected OR of 2.5 was based on the OR from the Wessels et al. study [5] because it was the only study to report an OR for this association. We were unable to calculate an OR for the Dervieux et al. study [3] because the outcome in that study was a continuous outcome (physician's assessment of response) rather than a categorical outcome (low vs high DAS28), as in our study [3]. Given these values, the FPRP was 0.33 and 0.30 for the MTX monotherapy and the all MTX groups, respectively.

We anticipate that others may disagree with our interpretation of the expected OR and/or the prior probability of an association between $A T I C$ and disease activity. Thus, the FPRP for a range of prior probabilities and expected ORs is provided in Table 5. Cutpoints for noteworthy FPRP values vary depending on statistical power and other study characteristics, but most studies cite FPRP thresholds ranging between 0.2 and 0.5 [16]. If future studies utilize the FPRP method, an increase in the prior probability of association between disease activity and ATIC rs4673993 (and SNPs in LD with rs4673993) may be justified. However, given the limitations of this study, we believe it is best to be conservative and await replication before making large adjustments.

The limitations of this study are its cross-sectional design and small sample size. We currently do not have data regarding the effect of MTX on disease activity over time, so we could not directly attribute differences in disease activity to differences in treatment response. Furthermore, with only 120 participants in the MTX monotherapy group, the power to detect an OR of 2.5 was only 0.20 . Thus, even though we did not find an association between disease activity and SNPs in ITPA and MTHFR, we could not exclude the possibility that modest associations exist. To increase our sample size, we performed a secondary analysis on the 262 participants on any MTX therapy. The increase in sample size improved the power to 0.82 , but the combined sample population was more heterogeneous.
Although still significant, the association between ATIC rs4673993 and disease activity was weaker in the combined cohort of 262 patients, compared with the primary cohort of participants on MTX monotherapy. Post hoc analyses showed that the association between $A T I C$ rs4673993 and disease activity was not significant among the 142 participants on combination therapy. Although we adjusted for the effects of anti-TNF agents and other DMARDs in multivariable analyses, residual confounding may have led to less significant associations between the genetic predictors and outcome in this cohort.

Future studies will include an analysis of RA patients newly started on MTX and followed over time. Other studies will also need to incorporate fine-mapping techniques to determine whether this SNP is responsible for differences in disease activity or whether it is in LD with another SNP that alters disease activity. Functional studies must be performed to confirm that changes in genotype translate into changes in enzyme level or activity.

Our study is intriguing because it suggests that ATIC polymorphisms may be associated with disease activity in RA patients on MTX. While it is interesting that the minor allele of ATIC rs4673993 is associated with low disease activity in this cohort, these results must be replicated in a larger cohort with sufficient power to detect modest effects.

\section{Rheumatology key messages}

- ATIC SNPs may be associated with disease activity in RA patients on MTX.

- Larger studies are needed to replicate reported associations between ATIC SNPS and MTX treatment response.

\section{Acknowledgements}

We would like to thank Robert Plenge for his advice regarding analysis of genetic data. We also thank the BRASS participants and study staff for making this study possible.

Funding: This work was supported by the National Institutes of Health [grant numbers R01 AR49880, P60 AR047782 and K24 AR0524-01 to E.W.K] and, Millenium Pharmaceuticals, Biogen Idec and Crescendo Bioscience. National Institutes of Health grant K30 RR022292-07 also provided support for Y.C.L.'s educational programme.

Disclosure statement: N.A.S. has received grant support from Amgen, Biogen Idec, Bristol-Myers Squibb foundation and Millenium Pharmaceutical. M.E.W. has received grant support funding for the BRASS Registry from Biogen/Idec, Millenium Pharmaceutical and Cresendo and is a consultant for Biogen/Idec, Millenium Pharmaceutical, Cresendo and Cypress Pharmaceuticals. All other authors have declared no conflicts of interest.

\section{References}

1 Weinblatt ME, Kaplan H, Germain BF et al. Methotrexate in rheumatoid arthritis. A five-year prospective multicenter study. Arthritis Rheum 1994;37:1492-8.

2 Kinder AJ, Hassell AB, Brand J, Brownfield A, Grove M, Shadforth MF. The treatment of inflammatory arthritis with methotrexate in clinical practice: treatment duration and incidence of adverse drug reactions. Rheumatology 2005;44:61-6.

3 Dervieux T, Furst D, Lein DO et al. Polyglutamation of methotrexate with common polymorphisms in reduced folate carrier, aminoimidazole carboxamide ribonucleotide transformylase, and thymidylate synthase are associated with methotrexate effects in rheumatoid arthritis. Arthritis Rheum 2004;50:2766-74.

4 Wessels JA, Kooloos WM, De Jonge $\mathrm{R}$ et al. Relationship between genetic variants in the adenosine pathway and outcome of methotrexate treatment in patients with recent-onset rheumatoid arthritis. Arthritis Rheum 2006;54:2830-9.

5 Wessels JA, van der Kooij SM, le Cessie S et al. A clinical pharmacogenetic model to predict the efficacy of methotrexate monotherapy in recent-onset rheumatoid arthritis. Arthritis Rheum 2007;56:1765-75. 
6 Dervieux T, Greenstein N, Kremer J. Pharmacogenomic and metabolic biomarkers in the folate pathway and their association with methotrexate effects during dosage escalation in rheumatoid arthritis. Arthritis Rheum 2006;54:3095-103.

7 Wessels JA, de Vries-Bouwstra JK, Heijmans BT et al. Efficacy and toxicity of methotrexate in early rheumatoid arthritis are associated with single-nucleotide polymorphisms in genes coding for folate pathway enzymes. Arthritis Rheum 2006;54:1087-95.

8 Kurzawski M, Pawlik A, Safranow K, Herczynska M,Drozdzik M. 677C $>$ T and 1298 A $>C$ MTHFR polymorphisms affect methotrexate treatment outcome in rheumatoid arthritis. Pharmacogenomics 2007;8:1551-9.

9 Karlson EW, Chibnik LB, Cui J et al. Associations between human leukocyte antigen, PTPN22, CTLA4 genotypes and rheumatoid arthritis phenotypes of autoantibody status, age at diagnosis and erosions in a large cohort study. Ann Rheum Dis 2008;67:358-63.

10 Anderson JJ, Wells G, Verhoeven AC, Felson DT. Factors predicting response to treatment in rheumatoid arthritis: the importance of disease duration. Arthritis Rheum 2000;43:22-9.

11 Plenge RM, Cotsapas C, Davies $L$ et al. Two independent alleles at 6q23 associated with risk of rheumatoid arthritis. Nat Genet 2007;39:1477-82.

12 Drozdzik M, Rudas T, Pawlik A et al. The effect of $3435 \mathrm{C}>\mathrm{T}$ MDR1 gene polymorphism on rheumatoid arthritis treatment with disease-modifying antirheumatic drugs. Eur J Clin Pharmacol 2006;62:933-7.

13 Takatori $\mathrm{R}$, Takahashi KA, Tokunaga $\mathrm{D}$ et al. ABCB1 C3435T polymorphism influences methotrexate sensitivity in rheumatoid arthritis patients. Clin Exp Rheumatol 2006;24:546-54.
14 Fabris M, Tolusso B, Di Poi E Assaloni R, Sinigaglia L, Ferraccioli G. Tumor necrosis factor-alpha receptor II polymorphism in patients from southern Europe with mildmoderate and severe rheumatoid arthritis. J Rheumatol 2002;29:1847-50.

15 Van Gestel AM, Haagsma CJ, Van Riel PLCM. Validation of rheumatoid arthritis improvement criteria that include simplified joint counts. Arthritis Rheum 1998;41:1845-50.

16 Wacholder S, Chanock S, Garcia-Closas M, El Ghormli L, Rothman N. Assessing the probability that a positive report is false: an approach for molecular epidemiology studies. J Natl Cancer Inst 2004;96:434-42.

$17 \mathrm{Li} \mathrm{C}$, Zhao H, Hu Z et al. Genetic variants and haplotypes of the caspase-8 and caspase-10 genes contribute to susceptibility to cutaneous melanoma. Hum Mutat 2008;29:1443-51.

18 Kelemen LE, Sellers TA, Schildkraut JM et al. Genetic variation in the one-carbon transfer pathway and ovarian cancer risk. Cancer Res 2008;68:2498-506.

19 Dossus L, McKay JD, Canzian F et al. Polymorphisms of genes coding for ghrelin and its receptor in relation to anthropometry, circulating levels of IGF-I and IGFBP-3, and breast cancer risk: a case-control study nested within the European Prospective Investigation into Cancer and Nutrition (EPIC). Carcinogenesis 2008;29: 1360-6.

20 Verheus M, McKay JD, Kaaks $\mathrm{R}$ et al. Common genetic variation in the IGF-1 gene, serum IGF-I levels and breast density. Breast Cancer Res Treat 2008;112: 109-22.

21 Mattick JS, Makunin IV. Non-coding RNA. Hum Mol Genet 2006;15:R17-29. 